\title{
About periodicity of impulsive evolution equations through fixed point theory
}

Jin Liang ${ }^{1 *}$, Ti-Jun Xiao ${ }^{2}$ and He Yang ${ }^{1}$

\section{"Correspondence:} jinliang@sjtu.edu.cn

'Department of Mathematics,

Shanghai Jiao Tong University,

Shanghai, 200240, P.R. China

Full list of author information is

available at the end of the article

\begin{abstract}
By processing the problem through fixed point theory and propagator theory, we investigate the periodicity of solutions to a class of impulsive evolution equations in Hilbert spaces and establish some existence theorems for periodic solutions. Moreover, the asymptotic stability of periodic solutions is obtained under suitable conditions. As one will see, the concept of an impulsive propagator is introduced for the first time in the paper.
\end{abstract}

MSC: 47D06; 47H10; 34G10; 34G20; 35Q99; 35K90; 35B40

Keywords: fixed point theorem; periodic solution; propagator; impulsive evolution equation; asymptotic stability; analytic semigroup

\section{Introduction}

It is well known that fixed point theorems play key roles in obtaining the existence of solutions, positive solution, periodic solutions, and almost periodic solutions to various equations or systems. There are many research publications in this area; for example, see [1-27] and the references therein for interesting results on this issue.

In this paper, by using the fixed point theory and propagator theory, we study the existence and asymptotic stability of periodic solutions for the following impulsive evolution equation in a Hilbert space $H$ :

$$
\left\{\begin{array}{l}
x^{\prime}(t)+A x(t)=f(t, x(t)), \quad t \in \mathbb{R}^{+}, t \neq t_{k}, \\
\left.\Delta x\right|_{t=t_{k}}=y_{k}-a_{k} x\left(t_{k}\right), \quad k=1,2, \ldots
\end{array}\right.
$$

where $A: D(A) \subset H \rightarrow H$ is a unbounded closed linear operator, $f(t, u): \mathbb{R}^{+} \times H \rightarrow H$ is a nonlinear mapping and it is $T$-periodic in $t, 0<t_{1}<t_{2}<\cdots<t_{m}<T$,

$$
t_{m+k}=t_{k}+T, \quad k \geq 1,
$$

$T>0$ is a fixed number and $m \in \mathbb{N}$ denotes the number of impulsive points between 0 and $T$,

$$
\left.\Delta u\right|_{t=t_{k}}=u\left(t_{k}^{+}\right)-u\left(t_{k}^{-}\right),
$$

(c) 2015 Liang et al. This article is distributed under the terms of the Creative Commons Attribution 4.0 International License (http://creativecommons.org/licenses/by/4.0/), which permits unrestricted use, distribution, and reproduction in any medium, provided you give appropriate credit to the original author(s) and the source, provide a link to the Creative Commons license, and indicate if changes were made. 
$u\left(t_{k}^{+}\right)$and $u\left(t_{k}^{-}\right)$represent the right and left limits of $u(t)$ at $t=t_{k}$, respectively, $a_{k} \in \mathbb{R}$, $y_{k} \in H(k=1,2, \ldots)$ satisfy

$$
a_{k+m}=a_{k}, \quad y_{k+m}=y_{k} .
$$

In the last few decades, the theory of impulsive differential equations has been largely developed. For the earlier results, we refer the reader to the monographs of Lakshmikantham et al. [13] and Benchohra et al. [3], the papers of Ahmed [28-30] and Liu [20], and the references cited therein. For the recent results, we refer the reader to, e.g., $[1,16,17,21$, $23,31]$ and the references therein.

On the other hand, the existence of periodic solutions or almost periodic solutions of evolution equations has been investigated by many authors (cf., e.g., $[2,4,5,9-11,14$, $15,17-19,24])$. One can see that all these studies are based on the fixed point theory. For example, in $[14,15]$, under the spectral separation condition of selfadjoint operators, Li obtained some existence and uniqueness results for periodic solutions to semilinear evolution equations in Hilbert spaces by using fixed point theorems. Moreover, we can find useful information on the study of almost periodicity of evolution equations with the help of fixed point theory from Cuevas et al. [4, 5], Diagana [9-11], Mophou [24] and references cited there.

Although there have been many papers on periodic solutions of periodic system in finite or infinite dimensional spaces, to our knowledge,impulsive periodic systems in infinite dimensional spaces (with unbounded operators) have been seldom investigated. Recently, Liang et al. (see [17]) studied a class of semilinear impulsive evolution equations with delay in Banach spaces. They proved the existence of $T$-periodic solutions by using Horn's fixed point theorem.

In this paper, we will use a different method, which is based on fixed point theorems and evolution operators, to study $T$-periodic solutions of (1.1). First of all, we introduce a new concept of impulsive propagator, corresponding to the linear impulsive evolution equation, and then we introduce a suitable $T$-periodic solution operator of (1.1). Second, we overcome some difficulties to show the existence and uniqueness of periodic solutions to (1.1) by using Schauder's fixed point theorem. Finally, we present the global asymptotic stability result for (1.1). Particularly, our discussion is made in a framework of Hilbert spaces, which enables us to obtain the existence theorems for strong $T$-periodic solutions of (1.1).

The rest of this paper is organized as follows. In Section 2, we introduce the impulsive propagator and then prove the existence of $T$-periodic solutions for the linear impulsive evolution equations. In Section 3, the existence and asymptotic stability theorems for $T$ periodic solutions to (1.1) are obtained. An example is given in Section 4 to illustrate the applicability of our results.

\section{Impulsive propagator and linear impulsive periodic systems}

Let $(H,(\cdot, \cdot))$ be a Hilbert space, $A: D(A) \subset H \rightarrow H$ be a positive definite selfadjoint operator and the embedding $D(A) \hookrightarrow H$ be compact. Then the spectrum of $A$ consists of real eigenvalues $\mu_{i}(i=1,2, \ldots)$, and

$$
\mu_{1}<\mu_{2}<\cdots<\mu_{n}<\cdots .
$$


By the positive definite property of $A$, the first eigenvalue $\mu_{1}>0$. It is known from [12, 25] that $A$ generates a compact and exponentially stable analytic semigroup $S(t)(t \geq 0)$ in $H$, and

$$
\|S(t)\| \leq e^{-\mu_{1} t}, \quad t \geq 0 .
$$

From $[12,25]$, we also know that for any $\sigma>0, A^{-\sigma}$ is defined by

$$
A^{-\sigma}=\frac{1}{\Gamma(\sigma)} \int_{0}^{\infty} t^{\sigma-1} S(t) d t
$$

and $A^{\sigma}$ is defined by

$$
A^{\sigma}=\left(A^{-\sigma}\right)^{-1}, \quad D\left(A^{\sigma}\right)=A^{-\sigma}(H)
$$

Let $H_{\sigma}$ be the Hilbert space $\left(D\left(A^{\sigma}\right),\langle\cdot, \cdot\rangle_{\sigma}\right)$, where

$$
\langle\cdot, \cdot\rangle_{\sigma}=\left(A^{\sigma} \cdot, A^{\sigma} \cdot\right) .
$$

Particularly, we write $H_{0}=H$ and $H_{1}=D(A)$.

Consider the linear Cauchy problem

$$
\left\{\begin{array}{l}
x^{\prime}(t)+A x(t)=h(t), \quad t \geq 0, \\
x(0)=x_{0} .
\end{array}\right.
$$

When $x_{0} \in D(A)$ and $h \in C^{1}([0, \infty), H)$, the linear Cauchy problem (2.1) has a classical solution $x \in C^{1}([0, \infty), H) \cap C\left([0, \infty), H_{1}\right)$ expressed by

$$
x(t)=S(t) x_{0}+\int_{0}^{t} S(t-s) h(s) d s
$$

When $x_{0} \in H$ and $h \in L_{\mathrm{loc}}^{1}([0, \infty), H)$, the function $x \in C([0, \infty), H)$ given by (2.2) is a mild solution of the linear Cauchy problem (2.1). If the function $x \in W_{\mathrm{loc}}^{1,1}([0, \infty), H) \cap$ $L_{\text {loc }}^{1}\left([0, \infty), H_{1}\right)$, then it is a strong solution of the linear Cauchy problem (2.1) (cf., e.g., [26, 27]).

Let $\widetilde{D}=\left\{t_{1}, t_{2}, \ldots, t_{m}\right\} \subset[0, T]$. Write

$$
\begin{aligned}
\operatorname{PC}([0, T], H):= & \{x:[0, T] \rightarrow H ; x \text { is continuous at } t \in[0, T] \backslash \widetilde{D}, \\
& x \text { is continuous from left and has right hand limits at } t \in \widetilde{D}\}
\end{aligned}
$$

and

$$
\begin{aligned}
\mathrm{PC}_{T}\left(\mathbb{R}^{+}, H\right):= & \left\{x: \mathbb{R}^{+} \rightarrow H ; x \text { is continuous at } t \in \mathbb{R}^{+} \backslash\left\{t_{1}, t_{2}, \ldots, t_{k}, \ldots\right\},\right. \\
& x \text { is continuous from left and has right hand limits at } t_{k}, \\
& \left.k=1,2, \ldots, x(t+T)=x(t) \text { for } t \in \mathbb{R}^{+}\right\} .
\end{aligned}
$$


It is clear that the restriction of $\mathrm{PC}_{T}\left(\mathbb{R}^{+}, H\right)$ on $[0, T]$ is $\mathrm{PC}([0, T], H)$. Set

$$
\|x\|_{\mathrm{PC}}=\max \left\{\sup _{t \in[0, T]}\|x(t+0)\|, \sup _{t \in[0, T]}\|x(t-0)\|\right\} .
$$

Then $\mathrm{PC}([0, T], H)\left(\right.$ or $\left.\mathrm{PC}_{T}\left(\mathbb{R}^{+}, H\right)\right)$ is a Banach space endowed with the norm $\|\cdot\|_{\mathrm{PC}}$.

Definition 2.1 Let $V(\cdot, \cdot): \Lambda \rightarrow \mathcal{L}_{b}(H)$ be denoted by

$$
V(t, \theta)=\prod_{\theta<t_{k}<t}\left(1-a_{k}\right) S(t-\theta)
$$

where $\Lambda:=\{(t, \theta) \mid 0 \leq \theta \leq t<\infty\}$. Then $\{V(t, \theta) \mid(t, \theta) \in \Lambda\}$ is called an impulse propagator associated with $\left\{a_{k}, t_{k}\right\}_{k=1}^{\infty}$.

Lemma 2.2 Impulsive propagator $\{V(t, \theta) \mid(t, \theta) \in \Lambda\}$ has the following properties:

(1) For $0 \leq \theta \leq t \leq T, V(t, \theta) \in \mathcal{L}_{b}(H)$, i.e.,

$$
\|V(t, \theta)\| \leq \prod_{k=1}^{m}\left|1-a_{k}\right| e^{-\mu_{1}(t-\theta)} .
$$

Particularly, if $0<a_{k}<1, k=1,2, \ldots, m$, then

$$
\|V(t, \theta)\|<e^{-\mu_{1}(t-\theta)} \leq 1
$$

(2) For $0 \leq \theta<r<t<\infty, r \neq t_{k}, k=1,2, \ldots, m$,

$$
V(t, \theta)=V(t, r) \cdot V(r, \theta)
$$

(3) For $0 \leq \theta \leq t<\infty$ and $N \in \mathbb{Z}_{0}^{+}$,

(i) $V(t+N T, \theta+N T)=V(t, \theta)$;

(ii) $V(t+N T, \theta)=V(t, \theta)[V(T, 0)]^{N}$;

(iii) $V(t+T, \theta)=V(t, 0) V(T, \theta)$.

(4) If $S(t)(t \geq 0)$ is a compact semigroup in $H$, then $V(t, \theta)$ is a compact operator for $0 \leq \theta<t \leq T$.

Proof By (2.3), we see easily that (1), (2), and (4) hold. Now, we prove that (3) also holds.

For any $0 \leq \theta \leq t<\infty$ and $N \in \mathbb{Z}_{0}^{+}$, by (2.3), we have

$$
\begin{aligned}
V(t+N T, \theta+N T) & =\prod_{\theta+N T<t_{k}<t+N T}\left(1-a_{k}\right) S(t+N T-\theta-N T) \\
& =\prod_{\theta<t_{k}<t}\left(1-a_{k}\right) S(t-\theta) \\
& =V(t, \theta)
\end{aligned}
$$


and

$$
\begin{aligned}
V(t+N T, \theta) & =\prod_{\theta<t_{k}<t+N T}\left(1-a_{k}\right) S(t+N T-\theta) \\
& =\prod_{\theta<t_{k}<t}\left(1-a_{k}\right) \prod_{t<t_{k}<t+N T}\left(1-a_{k}\right) S(t-\theta) S(N T) \\
& =V(t, \theta) \prod_{t<t_{k}<t+N T}\left(1-a_{k}\right) S(N T) \\
& =V(t, \theta)[V(T, 0)]^{N} .
\end{aligned}
$$

It implies that (i) and (ii) hold. Moreover,

$$
\begin{aligned}
V(t+T, \theta) & =\prod_{\theta<t_{k}<t+T}\left(1-a_{k}\right) S(t+T-\theta) \\
& =\prod_{\theta<t_{k}<T}\left(1-a_{k}\right) \prod_{T<t_{k}<t+T}\left(1-a_{k}\right) S(t) S(T-\theta) \\
& =V(T, \theta) \prod_{0<t_{k}<t}\left(1-a_{k}\right) S(t) \\
& =V(t, 0) V(T, \theta) .
\end{aligned}
$$

This implies that (iii) holds. By (ii) and (iii), we also get

$$
V(t+T, \theta)=V(t, \theta) V(T, 0)=V(t, 0) V(T, \theta) .
$$

This completes the proof of Lemma 2.2.

Let $h \in \mathrm{PC}_{T}\left(\mathbb{R}^{+}, H\right), a_{k} \in \mathbb{R}$, and $y_{k} \in H, k=1,2, \ldots$ We look at the existence of $T$ periodic solutions for the linear impulsive evolution equation

$$
\left\{\begin{array}{l}
x^{\prime}(t)+A x(t)=h(t), \quad t \in \mathbb{R}^{+}, t \neq t_{k}, \\
\left.\Delta x\right|_{t=t_{k}}=y_{k}-a_{k} x\left(t_{k}\right), \quad k=1,2, \ldots
\end{array}\right.
$$

For (2.4), we have the following result.

Lemma 2.3 Let $A: D(A) \subset H \rightarrow H$ be a positive definite selfadjoint operator in $H$ and the embedding $D(A) \hookrightarrow H$ be compact. If $h \in \mathrm{PC}_{T}\left(\mathbb{R}^{+}, H\right), a_{k} \in \mathbb{R}$, and $y_{k} \in H_{\frac{1}{2}}, k=1,2, \ldots$, then the linear impulsive evolution equation (2.4) has a unique T-periodic mild solution $x:=\mathcal{A} h \in \mathrm{PC}_{T}\left(\mathbb{R}^{+}, H\right)$ provided that $0<a_{k}<1, k=1,2, \ldots, m$, and $x \in W^{1,2}([0, T], H) \cap$ $L^{2}\left([0, T], H_{1}\right)$ is a strong solution. Moreover, $\mathcal{A}: \mathrm{PC}_{T}\left(\mathbb{R}^{+}, H\right) \rightarrow \mathrm{PC}_{T}\left(\mathbb{R}^{+}, H\right)$ is a compact operator. 
Proof Letting $h \in \mathrm{PC}([0, T], H)$, we first consider the Cauchy problem for linear impulsive evolution equations

$$
\left\{\begin{array}{l}
x^{\prime}(t)+A x(t)=h(t), \quad t \in[0, T], t \neq t_{k}, \\
\left.\Delta x\right|_{t=t_{k}}=y_{k}-a_{k} x\left(t_{k}\right), \quad k=1,2, \ldots, m, \\
x(0)=x_{0} .
\end{array}\right.
$$

Let $t_{0}=0, t_{m+1}=T, a_{0}=0$, and $y_{0}=0$. Then, for any $t \in\left(t_{k}, t_{k+1}\right], k=0,1,2, \ldots, m$, the Cauchy problem (2.5) becomes of the following form:

$$
\left\{\begin{array}{l}
x^{\prime}(t)+A x(t)=h(t), \quad t \in\left(t_{k}, t_{k+1}\right] \\
x\left(t_{k}^{+}\right)=\left(1-a_{k}\right) x\left(t_{k}\right)+y_{k}
\end{array}\right.
$$

It follows from $[12,27]$ that the problem $(2.6)$ has a unique mild solution expressed by

$$
x(t)=S\left(t-t_{k}\right)\left[\left(1-a_{k}\right) x\left(t_{k}\right)+y_{k}\right]+\int_{t_{k}}^{t} S(t-s) h(s) d s, \quad t \in\left(t_{k}, t_{k+1}\right] .
$$

Iterating successively in the equality above with $x\left(t_{n}\right), n=k, k-1, \ldots, 2,1,0$, we infer that

$$
x(t)=V(t, 0) x_{0}+\int_{0}^{t} V(t, \theta) h(\theta) d \theta+\sum_{0 \leq t_{k}<t} V\left(t, t_{k}^{+}\right) y_{k}, \quad t \in[0, T] .
$$

In view of the maximal regularity of linear evolution equations with positive definite operators in Hilbert spaces ([25], Chapter II, Theorem 3.3), we see that for $x_{0} \in H_{\frac{1}{2}}$, the mild solution of the linear Cauchy problem (2.1) satisfies

$$
x \in W^{1,2}([0, a], H) \cap L^{2}\left([0, a], H_{1}\right) \cap C\left([0, a], H_{\frac{1}{2}}\right),
$$

where $a>0$ is a fixed constant. Since $y_{k} \in H_{\frac{1}{2}}, k=1,2, \ldots, m$, we deduce by using (2.8) interval by interval from $\left[0, t_{1}\right]$ to $\left(t_{m}, T\right]$ that

$$
x \in W^{1,2}([0, T], H) \cap L^{2}\left([0, T], H_{1}\right) \cap \operatorname{PC}\left([0, T], H_{\frac{1}{2}}\right),
$$

where $x$ is the mild solution of the Cauchy problem (2.5).

On the other hand, if $x \in \mathrm{PC}_{T}\left(\mathbb{R}^{+}, H\right)$ is a $T$-periodic mild solution of the linear impulsive evolution equation (2.4), then $x$ is a mild solution of the Cauchy problem (2.5), which satisfies

$$
x_{0}:=x(0)=x(T) .
$$

By Lemma 2.2,

$$
\frac{1}{T} \sum_{i=1}^{m} \ln \left(1-a_{k}\right)<\mu_{1}
$$


Therefore, $I-V(T, 0)$ has a bounded inverse operator $(I-V(T, 0))^{-1}$. Hence, for

$$
x_{0}=(I-V(T, 0))^{-1}\left[\int_{0}^{T} V(T, \theta) h(\theta) d \theta+\sum_{k=1}^{m} V\left(T, t_{k}^{+}\right) y_{k}\right] \text {, }
$$

the Cauchy problem (2.5) has a unique mild solution $x$ given by (2.7) with

$$
x(T)=x(0)=x_{0} .
$$

Moreover, for any $t \geq 0$, we have

$$
\begin{aligned}
x(t)= & V(t, 0) x(0)+\int_{0}^{t} V(t, \theta) h(\theta) d \theta+\sum_{0 \leq t_{k} \leq t} V\left(t, t_{k}^{+}\right) y_{k} \\
= & V(t, 0) x(T)+\int_{0}^{t} V(t, \theta) h(\theta) d \theta+\sum_{0 \leq t_{k} \leq t} V\left(t, t_{k}^{+}\right) y_{k} \\
= & V(t, 0)\left[V(T, 0) x(0)+\int_{0}^{T} V(T, \theta) h(\theta) d \theta+\sum_{0 \leq t_{k}<T} V\left(T, t_{k}^{+}\right) y_{k}\right] \\
& +\int_{0}^{t} V(t, \theta) h(\theta) d \theta+\sum_{0 \leq t_{k} \leq t} V\left(t, t_{k}^{+}\right) y_{k} \\
= & V(t, 0) V(T, 0) x(0)+V(t, 0) \int_{0}^{T} V(T, \theta) h(\theta) d \theta+\int_{T}^{t+T} V(t+T, \theta) h(\theta) d \theta \\
& +V(t, 0) \sum_{0 \leq t_{k}<T} V\left(T, t_{k}^{+}\right) y_{k}+\sum_{T \leq t_{k} \leq t+T} V\left(t+T, t_{k}^{+}\right) y_{k} \\
= & V(t+T, 0) x(0)+\int_{0}^{t+T} V(t+T, \theta) h(\theta) d \theta+\sum_{0 \leq t_{k}<t+T} V\left(t+T, t_{k}^{+}\right) y_{k} \\
= & x(t+T) .
\end{aligned}
$$

This implies that the $T$-periodic extension of $x$ on $\mathbb{R}^{+}$is the unique $T$-periodic mild solution of the linear impulsive evolution equation (2.4). Moreover, we can see that $x \in W^{1,2}([0, T], H) \cap L^{2}\left([0, T], H_{1}\right)$ is a strong $T$-periodic solution of the linear impulsive evolution equation (2.4) and

$$
\begin{aligned}
x(t)= & V(t, 0)(I-V(T, 0))^{-1}\left[\int_{0}^{T} V(T, \theta) h(\theta) d \theta+\sum_{k=1}^{m} V\left(T, t_{k}^{+}\right) y_{k}\right] \\
& +\int_{0}^{t} V(t, \theta) h(\theta) d \theta+\sum_{0 \leq t_{k}<t} V\left(t, t_{k}^{+}\right) y_{k} \\
:= & (\mathcal{A} h)(t), \quad t \in[0, T] .
\end{aligned}
$$

Let

$$
0 \leq \sigma<\frac{1}{2}, \quad \lambda \in\left(0, \frac{1}{2}-\sigma\right)
$$


Then the solution operator

$$
\mathcal{A}: \mathrm{PC}([0, T], H) \rightarrow \mathrm{PC}^{\lambda}\left([0, T], H_{\sigma}\right)
$$

is a continuous linear operator by [2], Lemma 2.1 and Corollary 2.2. Thus, Aszela-Ascoli's theorem shows that the embedding

$$
\mathrm{PC}^{\lambda}\left([0, T], H_{\sigma}\right) \hookrightarrow \mathrm{PC}([0, T], H)
$$

is compact. Thus, $\mathcal{A}: \mathrm{PC}_{T}\left(\mathbb{R}^{+}, H\right) \rightarrow \mathrm{PC}_{T}\left(\mathbb{R}^{+}, H\right)$ is a compact linear operator.

\section{Main results}

Next we always assume that $a_{k} \in(0,1)$ and $y_{k} \in H_{\frac{1}{2}}, k=1,2, \ldots, m$. First, we consider the following Cauchy problem:

$$
\left\{\begin{array}{l}
x^{\prime}(t)+A x(t)=f(t, x(t)), \quad t \geq 0, t \neq t_{k}, \\
\left.\Delta x\right|_{t=t_{k}}=y_{k}-a_{k} x\left(t_{k}\right), \quad k=1,2, \ldots, \\
x(0)=x_{0} \in H .
\end{array}\right.
$$

For the Cauchy problem (3.1), we have the following uniqueness theorem.

Theorem 3.1 Let $-A$ be the infinitesimal generator of a $C_{0}$-semigroup $S(t)(t \geq 0)$. Assume that

(H1) There exists a constant $C>0$ such that

$$
\|f(t, u)-f(t, v)\| \leq C\|u-v\|, \quad t \geq 0, u, v \in H .
$$

Then the Cauchy problem (3.1) has a unique strong solution

$$
x \in W_{\mathrm{loc}}^{1,2}((0, \infty), H) \cap L_{\mathrm{loc}}^{2}\left((0, \infty), H_{1}\right) \cap \mathrm{PC}([0, \infty), H),
$$

and

$$
x(t)=V(t, 0) x_{0}+\int_{0}^{t} V(t, \theta) f(\theta, x(\theta)) d \theta+\sum_{0 \leq t_{k}<t} V\left(t, t_{k}^{+}\right) y_{k}, \quad t \geq 0 .
$$

Proof For $t \in\left[0, t_{1}\right]$, the Cauchy problem (3.1) is in the following form:

$$
\left\{\begin{array}{l}
x^{\prime}(t)+A x(t)=f(t, x(t)), \quad t \in\left[0, t_{1}\right] \\
x(0)=x_{0} .
\end{array}\right.
$$

By [26], Chapter 6, Theorem 1.2, the Cauchy problem (3.2) has a unique global mild solution $\bar{x} \in C\left(\left[0, t_{1}\right], H\right)$, and

$$
\bar{x}(t)=S(t) x_{0}+\int_{0}^{t} S(t-s) f(s, x(s)) d s, \quad t \in\left[0, t_{1}\right]
$$


For $t \in\left(t_{1}, t_{2}\right]$, the Cauchy problem (3.1) is of the following form:

$$
\left\{\begin{array}{l}
x^{\prime}(t)+A x(t)=f(t, x(t)), \quad t \in\left(t_{1}, t_{2}\right] \\
x\left(t_{1}^{+}\right)=\left(1-a_{1}\right) \bar{x}\left(t_{1}\right)+y_{1}
\end{array}\right.
$$

By (3.3), the Cauchy problem (3.4) has a unique mild solution

$$
\begin{aligned}
x_{1}(t)= & S\left(t-t_{1}\right)\left[\left(1-a_{1}\right) \bar{x}\left(t_{1}\right)+y_{1}\right]+\int_{t_{1}}^{t} S(t-s) f(s, x(s)) d s \\
= & \left(1-a_{1}\right) S(t) x_{0}+\int_{0}^{t_{1}}\left(1-a_{1}\right) S(t-s) f(s, x(s)) d s \\
& +\int_{t_{1}}^{t} S(t-s) f(s, x(s)) d s+S\left(t-t_{1}\right) y_{1} .
\end{aligned}
$$

Doing this interval by interval, we obtain

$$
x(t)=V(t, 0) x_{0}+\int_{0}^{t} V(t, \theta) f(\theta, x(\theta)) d \theta+\sum_{0 \leq t_{k}<t} V\left(t, t_{k}^{+}\right) y_{k}, \quad t \geq 0 .
$$

Let $x$ be the mild solution of Cauchy problem (3.1). Then we can deduce that

$$
x \in W_{\text {loc }}^{1,2}((0, \infty), H) \cap L_{\text {loc }}^{2}\left((0, \infty), H_{1}\right),
$$

and it is a strong solution of the Cauchy problem (3.1).

Now, we can consider the existence, uniqueness, and asymptotic stability of $T$-periodic solutions for the impulsive evolution equation

$$
\left\{\begin{array}{l}
x^{\prime}(t)+A x(t)=f(t, x(t)), \quad t \in \mathbb{R}^{+}, t \neq t_{k} \\
\left.\Delta x\right|_{t=t_{k}}=y_{k}-a_{k} x\left(t_{k}\right), \quad k=1,2, \ldots
\end{array}\right.
$$

where $f: \mathbb{R}^{+} \times H \rightarrow H$ is continuous and $f(t, x)$ is $T$-periodic in $t$.

For the impulsive evolution equation (3.5), we have the following theorem.

Theorem 3.2 Let $A: D(A) \subset H \rightarrow H$ be a positive definite selfadjoint operator in $H$ and the embedding $D(A) \hookrightarrow H$ be compact. Assume that

(H2) There exist two constants $M_{1} \in\left(0, \mu_{1}\right)$ and $M_{2}>0$ such that

$$
\|f(t, u)\| \leq M_{1}\|u\|+M_{2}, \quad t \in \mathbb{R}^{+} .
$$

Then the impulsive evolution equation (3.5) has at least one strong T-periodic solution

$$
x \in W_{\text {loc }}^{1,2}\left(\mathbb{R}^{+}, H\right) \cap L_{\text {loc }}^{2}\left(\mathbb{R}^{+}, H_{1}\right) \cap \mathrm{PC}_{T}\left(\mathbb{R}^{+}, H\right) .
$$

Proof Set

$$
G(x)(t):=f(t, x(t)), \quad x \in \mathrm{PC}_{T}\left(\mathbb{R}^{+}, H\right), t \in \mathbb{R}^{+} .
$$


Then $G: \mathrm{PC}_{T}\left(\mathbb{R}^{+}, H\right) \rightarrow \mathrm{PC}_{T}\left(\mathbb{R}^{+}, H\right)$ is continuous and maps bounded sets in $\mathrm{PC}_{T}\left(\mathbb{R}^{+}, H\right)$ into bounded sets. By Lemma 2.3 and the fact that

$$
\mathcal{A}: \mathrm{PC}_{T}\left(\mathbb{R}^{+}, H\right) \rightarrow \mathrm{PC}_{T}\left(\mathbb{R}^{+}, H\right)
$$

is a compact operator, we see that the operator

$$
\Phi:=\mathcal{A} \circ G: \mathrm{PC}_{T}\left(\mathbb{R}^{+}, H\right) \rightarrow \mathrm{PC}_{T}\left(\mathbb{R}^{+}, H\right)
$$

is completely continuous. It is clear that $T$-periodic mild solutions of the impulsive evolution equation (3.5) are equivalent to fixed points of operator $\Phi$.

Choose

$$
r>\frac{M_{2}\left(1-e^{-\mu_{1} T}\right)+\mu_{1} \sum_{k=1}^{m}\left\|y_{k}\right\|}{\left(\mu_{1}-M_{1}\right)\left(1-e^{-\mu_{1} T}\right)}
$$

and let

$$
B_{r}:=\left\{x \in \mathrm{PC}_{T}\left(\mathbb{R}^{+}, H\right):\|x\|_{\mathrm{PC}} \leq r\right\} .
$$

Then, for every $x \in B_{r}$ and $t \in[0, T]$,

$$
\left\|(I-V(T, 0))^{-1}\right\|=\left\|\sum_{n=0}^{\infty}[V(T, 0)]^{n}\right\| \leq \sum_{n=0}^{\infty} \prod_{k=1}^{m}\left(1-a_{k}\right)^{n} e^{-n \mu_{1} T} \leq \frac{1}{1-e^{-\mu_{1} T}} .
$$

Hence,

$$
\begin{aligned}
\|(\Phi x)(t)\|= & \|\mathcal{A}(G(x))(t)\| \\
\leq & \left\|V(t, 0)(I-V(T, 0))^{-1}\left[\int_{0}^{T} V(T, \theta) f(\theta, x(\theta)) d \theta+\sum_{k=1}^{m} V\left(T, t_{k}^{+}\right) y_{k}\right]\right\| \\
& +\int_{0}^{t}\|V(t, \theta) f(\theta, x(\theta))\| d \theta+\sum_{0 \leq t_{k}<t}\left\|V\left(t, t_{k}^{+}\right) y_{k}\right\| \\
\leq & \frac{e^{-\mu_{1} t}}{1-e^{-\mu_{1} T}}\left[\int_{0}^{T} e^{-\mu_{1}(T-\theta)} d \theta\left(M_{1} r+M_{2}\right)+\sum_{k=1}^{m}\left\|y_{k}\right\|\right] \\
& +\frac{1-e^{-\mu_{1} t}}{\mu_{1}}\left(M_{1} r+M_{2}\right)+\sum_{k=1}^{m}\left\|y_{k}\right\| \\
\leq & \frac{M_{1} r+M_{2}}{\mu_{1}}+\left(\frac{e^{-\mu_{1} t}}{1-e^{-\mu_{1} T}}+1\right) \sum_{k=1}^{m}\left\|y_{k}\right\| \\
= & \frac{M_{1} r+M_{2}}{\mu_{1}}+\frac{\sum_{k=1}^{m}\left\|y_{k}\right\|}{1-e^{-\mu_{1} T}} .
\end{aligned}
$$

This means that $\|\Phi x\|_{\mathrm{PC}} \leq r$. So, $\Phi\left(B_{r}\right) \subset B_{r}$ is completely continuous. By Schauder's fixed point theorem, we know that $\Phi$ has at least one fixed point $\hat{x}$ in $B_{r}$. Therefore, $\hat{x}$ is a 
$T$-periodic mild solution of the impulsive evolution equation (3.5). By Lemma 2.3,

$$
\hat{x} \in W_{\mathrm{loc}}^{1,2}\left(\mathbb{R}^{+}, H\right) \cap L_{\mathrm{loc}}^{2}\left(\mathbb{R}^{+}, H_{1}\right) \cap \mathrm{PC}_{T}\left(\mathbb{R}^{+}, H\right)
$$

is also a strong $T$-periodic solution of the impulsive evolution equation (3.5).

For the impulsive evolution equation (3.5), we also have the following uniqueness and asymptotic stability theorem.

Theorem 3.3 Let $A: D(A) \subset H \rightarrow H$ be a positive definite selfadjoint operator in $H$ and the embedding $D(A) \hookrightarrow H$ is compact. Assume that

(H3) There exists a constant $0<M<\mu_{1}$ such that

$$
\|f(t, u)-f(t, v)\| \leq M\|u-v\|, \quad t \in \mathbb{R}^{+}, u, v \in H .
$$

Then the impulsive evolution equation (3.5) has a unique strong T-periodic solution which belongs to $W_{\text {loc }}^{1,2}\left(\mathbb{R}^{+}, H\right) \cap L_{\text {loc }}^{2}\left(\mathbb{R}^{+}, H_{1}\right)$ and it is globally asymptotically stable.

Proof Since the condition (H3) $\Rightarrow(\mathrm{H} 2)$ holds, by Theorem 3.2, the impulsive evolution equation (3.5) has at least one strong $T$-periodic solution which belongs to $W_{\text {loc }}^{1,2}\left(\mathbb{R}^{+}, H\right) \cap$ $L_{\text {loc }}^{2}\left(\mathbb{R}^{+}, H_{1}\right)$. Let $x_{1}, x_{2}$ be strong $T$-periodic solutions of the impulsive evolution equation (3.5). Then they are fixed points of the operator $\Phi$. By the definition of $\Phi$, we have

$$
\begin{aligned}
\left\|x_{1}(t)-x_{2}(t)\right\|= & \left\|\left(\Phi x_{1}\right)(t)-\left(\Phi x_{2}\right)(t)\right\| \\
\leq & \left\|V(t, 0)(I-V(T, 0))^{-1} \int_{0}^{T} V(T, \theta)\left[f\left(\theta, x_{1}(\theta)\right)-f\left(\theta, x_{2}(\theta)\right)\right] d \theta\right\| \\
& +\int_{0}^{t}\left\|V(t, \theta)\left[f\left(\theta, x_{1}(\theta)\right)-f\left(\theta, x_{2}(\theta)\right)\right]\right\| d \theta \\
\leq & \frac{e^{-\mu_{1} t}}{1-e^{-\mu_{1} T}} \int_{0}^{T} e^{-\mu_{1}(T-\theta)}\left\|f\left(\theta, x_{1}(\theta)\right)-f\left(\theta, x_{2}(\theta)\right)\right\| d \theta \\
& +\int_{0}^{t} e^{-\mu_{1}(t-\theta)}\left\|f\left(\theta, x_{1}(\theta)\right)-f\left(\theta, x_{2}(\theta)\right)\right\| d \theta .
\end{aligned}
$$

Therefore,

$$
\begin{aligned}
\left\|x_{1}-x_{2}\right\|_{\mathrm{PC}} & \leq\left[\frac{M e^{-\mu_{1} t}}{\mu_{1}}+\frac{M\left(1-e^{-\mu_{1} t}\right)}{\mu_{1}}\right] \cdot\left\|x_{1}-x_{2}\right\|_{\mathrm{PC}} \\
& =\frac{M}{\mu_{1}}\left\|x_{1}-x_{2}\right\|_{\mathrm{PC}} .
\end{aligned}
$$

Hence $x_{1} \equiv x_{2}$. Thus, the impulsive evolution equation (3.5) has a unique strong $T$ periodic solution.

Let $\hat{x} \in \mathrm{PC}_{T}\left(\mathbb{R}^{+}, H\right)$ be the unique strong $T$-periodic solution of the impulsive evolution equation (3.5). By Theorem 3.1, for any $x_{0} \in H$, the Cauchy problem (3.1) has a unique strong solution

$$
x(t):=x\left(t ; x_{0}\right) \in W_{\mathrm{loc}}^{1,2}((0, \infty), H) \cap L_{\mathrm{loc}}^{2}\left((0, \infty), H_{1}\right) \cap \mathrm{PC}([0, \infty), H) .
$$


Hence,

$$
\begin{aligned}
\|x(t)-\hat{x}(t)\| & \leq e^{-\mu_{1} t}\|x(0)-\hat{x}(0)\|+M \int_{0}^{t} e^{-\mu_{1}(t-s)}\|x(s)-\hat{x}(s)\| d s \\
& =e^{-\mu_{1} t}\|x(0)-\hat{x}(0)\|+M e^{-\mu_{1} t} \int_{0}^{t} e^{\mu_{1} s}\|x(s)-\hat{x}(s)\| d s, \quad t \geq 0 .
\end{aligned}
$$

Write $\rho(t):=e^{\mu_{1} t}\|x(t)-\hat{x}(t)\|$. Then, for $t \in[0, \infty)$, by the inequality above, we have

$$
\rho(t) \leq \rho(0)+M \int_{0}^{t} \rho(s) d s
$$

So

$$
e^{\mu_{1} t}\|x(t)-\hat{x}(t)\| \leq\|x(0)-\hat{x}(0)\| e^{M t}
$$

It follows that

$$
\|x(t)-\hat{x}(t)\| \leq\|x(0)-\hat{x}(0)\| e^{-\left(\mu_{1}-M\right) t} \rightarrow 0 \quad(t \rightarrow \infty) .
$$

Therefore, the strong $T$-periodic solution $\hat{x}$ of the impulsive evolution equation (3.5) is globally asymptotically stable and it exponentially attracts every solution of the corresponding Cauchy problem.

\section{Impulsive problem for a class of parabolic equations}

Consider time $2 \pi$-periodic solutions of the impulsive problem for the following parabolic equation:

$$
\left\{\begin{array}{l}
\frac{\partial}{\partial t} x(z, t)=\sum_{i, j=1}^{N} \frac{\partial}{\partial z_{i}}\left(\tau_{i j}(z) \frac{\partial x}{\partial z_{j}}\right)-\tau_{0}(z) x(z, t)+f(z, t, x(z, t)), \\
\quad z \in \Omega, t \in \mathbb{R}^{+} \backslash\left\{\frac{k}{2} \pi ; k=1,2, \ldots\right\}, \\
\Delta x\left(z, t_{k}\right)=y_{k}-a_{k} x\left(z, t_{k}\right), \quad z \in \Omega, t_{k}=\frac{k}{2} \pi, k=1,2, \ldots, \\
\left.x(z, t)\right|_{z \in \partial \Omega}=0,
\end{array}\right.
$$

where $\Omega \subset \mathbb{R}^{N}$ is a bounded domain, whose boundary $\partial \Omega$ is sufficiently smooth, $f: \bar{\Omega} \times$ $\mathbb{R}^{+} \times \mathbb{R} \rightarrow \mathbb{R}$ is continuous and is $2 \pi$-periodic in the second variable.

We assume that the following conditions are satisfied.

(A1) $\tau_{i j} \in C^{1+\nu}(\bar{\Omega})(i, j=1,2, \ldots, N),\left[\tau_{i j}(z)\right]_{N \times N}$ is a positive definite symmetric matrix for $z \in \bar{\Omega}$ and there exists a constant $\gamma>0$ such that

$$
\sum_{i, j=1}^{N} \tau_{i j}(z) \zeta_{i} \zeta_{j} \geq \gamma|\zeta|^{2}, \quad \forall \zeta=\left(\zeta_{1}, \zeta_{2}, \ldots, \zeta_{N}\right) \in \mathbb{R}^{N}, z \in \bar{\Omega}
$$

(A2) $\tau_{0} \in C^{v}(\bar{\Omega})$ for some $v \in(0,1), \tau_{0}(z) \geq 0$ on $\bar{\Omega}$.

Furthermore, there exists a function $p:[0, \infty) \rightarrow[0, \infty)$ such that $f: \bar{\Omega} \times \mathbb{R}^{+} \times \mathbb{R} \rightarrow \mathbb{R}$ satisfies

$$
\left|f(z, t, \xi)-f\left(z^{\prime}, t^{\prime}, \xi^{\prime}\right)\right| \leq p(\eta)\left(\left|z-z^{\prime}\right|^{\nu}+\left|t-t^{\prime}\right|^{\frac{v}{2}}+\left|\xi-\xi^{\prime}\right|\right),
$$

for any $\eta>0$ and $(z, t, \xi),\left(z^{\prime}, t^{\prime}, \xi^{\prime}\right) \in \bar{\Omega} \times \mathbb{R}^{+} \times \bar{B}(\mathbb{R} ; 0, \eta)$, where $\bar{B}(\mathbb{R} ; 0, \eta)=\{\xi \in \mathbb{R}:|\xi| \leq \eta\}$. 
Take $H=L^{2}(\Omega)$. Define

$$
A x:=\sum_{i, j=1}^{N} \frac{\partial}{\partial z_{i}}\left(\tau_{i j}(z) \frac{\partial x}{\partial z_{j}}\right)-\tau_{0}(z) x, \quad D(A):=H^{2}(\Omega) \cap H_{0}^{1}(\Omega) .
$$

Then $A$ is a positive definite selfadjoint operator in $H$ and $D\left(A^{\frac{1}{2}}\right)=H_{0}^{1}(\Omega)$. So, $A$ generates a compact analytic semigroup $S(t)(t \geq 0)$ in $H$ which is exponentially stable. Let

$$
a_{k}:=\frac{k}{10}, \quad k=1,2,3,4
$$

with $a_{k+4}:=a_{k}$ and $y_{k} \in H_{0}^{1}(\Omega)$ with $y_{k+4}:=y_{k}$. It is obvious that

$$
t_{k+4}=t_{k}+2 \pi
$$

Let $\mu_{1}$ be the smallest eigenvalue of operator

$$
\sum_{i, j=1}^{N} \frac{\partial}{\partial z_{i}}\left(\tau_{i j}(z) \frac{\partial x}{\partial z_{j}}\right)-\tau_{0}(z)
$$

under the Dirichlet boundary condition $\left.x\right|_{\partial \Omega}=0$. Then $\mu_{1}>0$. Under the above assumptions, we have the following existence theorem.

\section{Theorem 4.1 Assume that}

(F1) There exist two constants $M_{1} \in\left(0, \mu_{1}\right)$ and $M_{2}>0$ such that

$$
|f(z, t, \xi)| \leq M_{1}|\xi|+M_{2}, \quad z \in \Omega, t \in \mathbb{R}^{+}, \xi \in \mathbb{R} .
$$

Then the impulsive boundary value problem (4.1) has a time $2 \pi$-periodic solution $x \in$ $C^{2+v, 1+\frac{v}{2}}\left(\bar{\Omega} \times \mathbb{R}^{+}\right)$.

Proof Define

$$
\begin{aligned}
& x(\cdot)(z):=x(z, \cdot), \\
& f(\cdot, x(\cdot))(z):=f(z, \cdot, x(z, \cdot)) .
\end{aligned}
$$

Then the impulsive boundary value problem (4.1) can be rewritten in the form of (3.5). By Theorem 3.2, the boundary value problem (4.1) has at least one strong time $2 \pi$-periodic solution

$$
x \in \mathrm{PC}_{2 \pi}\left(\mathbb{R}^{+}, H_{0}^{1}(\Omega)\right) \cap L_{\mathrm{loc}}^{2}\left(\mathbb{R}^{+}, H^{2}(\Omega)\right) \cap W_{\mathrm{loc}}^{1,2}\left(\mathbb{R}^{+}, L^{2}(\Omega)\right) .
$$

By the regularization method in [2], Lemma 4.2, we can prove that $x \in C^{2+v, 1+\frac{v}{2}}\left(\bar{\Omega} \times \mathbb{R}^{+}\right)$ is a classical time $2 \pi$-periodic solution of the boundary value problem (4.1). 


\section{Theorem 4.2 Assume that}

(F2) There exists a constant $M \in\left(0, \mu_{1}\right)$ such that

$$
\left|f\left(z, t, \xi_{1}\right)-f\left(z, t, \xi_{2}\right)\right| \leq M\left|\xi_{1}-\xi_{2}\right|, \quad z \in \Omega, t \in \mathbb{R}^{+}, \xi_{1}, \xi_{2} \in \mathbb{R}
$$

Then the boundary value problem (4.1) has a unique time $2 \pi$-periodic solution $x \in$ $C^{2+v, 1+\frac{v}{2}}\left(\bar{\Omega} \times \mathbb{R}^{+}\right)$, which exponentially attracts every solution of the boundary value problem (4.1) with initial value condition $x(z, 0)=x_{0}(z)$ in $L^{2}(\Omega)$.

Proof Clearly, the condition (F2) implies the conditions (H1) and (H3) hold. By Theorem 3.1, the boundary value problem (4.1) has a unique solution $x\left(z, t ; x_{0}\right) \in C^{2+v, 1+\frac{v}{2}}(\bar{\Omega} \times$ $(0, \infty)$ ), which satisfies the initial value condition $x(z, 0)=x_{0}(z)$. By Theorem 3.3, the boundary value problem (4.1) has a unique time $2 \pi$-periodic solution $\tilde{x} \in \mathrm{PC}_{2 \pi}((0, \infty)$, $\left.L^{2}(\Omega)\right) \cap C^{2+v, 1+\frac{v}{2}}\left(\bar{\Omega} \times \mathbb{R}^{+}\right)$, which exponentially attracts $x\left(z, t ; x_{0}\right)$ in $L^{2}(\Omega)$.

\section{Applications}

Example 5.1 We consider the following impulsive boundary value problem:

$$
\left\{\begin{array}{l}
\frac{\partial}{\partial t} x(z, t)=\sum_{i, j=1}^{N} \frac{\partial^{2} x}{\partial z_{i} \partial z_{j}}-x(z, t)+\frac{\mu_{1} \sin t}{2} \frac{|x(z, t)|}{1+|x(z, t)|}+\frac{2}{e^{z}}, \\
\quad z \in \Omega, t \in \mathbb{R}^{+} \backslash\left\{\frac{k}{2} \pi ; k=1,2, \ldots\right\}, \\
\Delta x\left(z, t_{k}\right)=y_{k}-a_{k} x\left(z, t_{k}\right), \quad z \in \Omega, t_{k}=\frac{k}{2} \pi, k=1,2, \ldots, \\
\left.x(z, t)\right|_{z \in \partial \Omega}=0,
\end{array}\right.
$$

where $\Omega \subset \mathbb{R}^{N}$ is a bounded domain whose boundary $\partial \Omega$ is sufficiently smooth,

$$
a_{k}:=\frac{k}{100}, \quad k=1,2,3,4
$$

with $a_{k+4}:=a_{k}, y_{k} \in H_{0}^{1}(\Omega)$ with $y_{k+4}:=y_{k}$, and $\mu_{1}$ is the smallest eigenvalue of operator $\sum_{i, j=1}^{N} \frac{\partial}{\partial z_{i}}\left(\tau_{i j}(z) \frac{\partial x}{\partial z_{j}}\right)-\tau_{0}(z)$ under the Dirichlet boundary condition $\left.x\right|_{\partial \Omega}=0$.

Let $H=L^{2}(\Omega), A$ be the operator as in (4.2), and

$$
f(z, t, x(z, t)):=\frac{\mu_{1} \sin t}{2} \frac{|x(z, t)|}{1+|x(z, t)|}+\frac{2}{e^{z}} .
$$

Then we have

$$
|f(z, t, x(z, t))| \leq\left|\frac{\mu_{1} \sin t}{2} \frac{|x(z, t)|}{1+|x(z, t)|}\right|+\left|\frac{2}{e^{z}}\right| \leq \frac{\mu_{1}}{2}|x(z, t)|+2
$$

and

$$
\begin{aligned}
\|f(t, x(t))\|_{2} & \leq\left(\int_{\Omega}\left|\frac{\mu_{1}}{2}\right| x(z, t)|+2|^{2} d z\right)^{\frac{1}{2}} \\
& \leq \frac{\mu_{1}}{2}\|x\|_{2}+2 .
\end{aligned}
$$

It implies that the conditions (F1) and (H2) hold. Hence the impulsive boundary value problem (5.1) can be rewritten into the abstract form (3.5). Thus, by Theorem 3.2, the 
problem (5.1) has at least one strong time $2 \pi$-periodic solution

$$
X \in \mathrm{PC}_{2 \pi}\left(\mathbb{R}^{+}, H_{0}^{1}(\Omega)\right) \cap L_{\mathrm{loc}}^{2}\left(\mathbb{R}^{+}, H^{2}(\Omega)\right) \cap W_{\mathrm{loc}}^{1,2}\left(\mathbb{R}^{+}, L^{2}(\Omega)\right) .
$$

By the same argument as in the proof of Theorem 4.1, we see that $x \in C^{2+v, 1+\frac{v}{2}}\left(\bar{\Omega} \times \mathbb{R}^{+}\right)$is a classical time $2 \pi$-periodic solution of the boundary value problem (5.1).

Example 5.2 We take a look at the following impulsive problem:

$$
\left\{\begin{array}{l}
\frac{\partial}{\partial t} x(z, t)=\sum_{i, j=1}^{N} \frac{\partial^{2} x}{\partial z_{i} \partial z_{j}}-x(z, t)+\frac{\mu_{1} \sin t}{3 e^{z}} \frac{|x(z, t)|}{1+|x(z, t)|}, \\
\quad z \in \Omega, t \in \mathbb{R}^{+} \backslash\left\{\frac{k}{2} \pi ; k=1,2, \ldots\right\}, \\
\Delta x\left(z, t_{k}\right)=y_{k}-a_{k} x\left(z, t_{k}\right), \quad z \in \Omega, t_{k}=\frac{k}{2} \pi, k=1,2, \ldots, \\
\left.x(z, t)\right|_{z \in \partial \Omega}=0,
\end{array}\right.
$$

where $\Omega, a_{k}, y_{k}$ are the same as in (4.1), and $\mu_{1}$ is a constant as in (5.1).

Let $H=L^{2}(\Omega)$, $A$ be the operator as in (4.2), and

$$
f(z, t, x(z, t)):=\frac{\mu_{1} \sin t}{3 e^{z}} \frac{|x(z, t)|}{1+|x(z, t)|} .
$$

Then

$$
\begin{aligned}
\left|f\left(z, t, x_{1}(z, t)\right)-f\left(z, t, x_{2}(z, t)\right)\right| & \leq\left|\frac{\mu_{1} \sin t}{3 e^{z}}\left(\frac{\left|x_{1}(z, t)\right|}{1+\left|x_{1}(z, t)\right|}-\frac{\left|x_{2}(z, t)\right|}{1+\left|x_{2}(z, t)\right|}\right)\right| \\
& \leq \frac{\mu_{1}}{3}\left|x_{1}(z, t)-x_{2}(z, t)\right|
\end{aligned}
$$

and

$$
\begin{aligned}
\left\|f\left(t, x_{1}(t)\right)-f\left(t, x_{2}(t)\right)\right\|_{2} & \leq\left(\int_{\Omega}\left|\frac{\mu_{1}}{3}\right| x_{1}(z, t)-x_{2}(z, t)||^{2} d z\right)^{\frac{1}{2}} \\
& \leq \frac{\mu_{1}}{3}\left\|x_{1}-x_{2}\right\|_{2} .
\end{aligned}
$$

It implies that the conditions (F2), (H1), and (H3) hold. Therefore, by Theorem 3.1, the problem (5.2) has a unique solution $x\left(z, t ; x_{0}\right) \in C^{2+v, 1+\frac{v}{2}}(\bar{\Omega} \times(0, \infty))$, which satisfies the initial value condition $x(z, 0)=x_{0}(z)$. Moreover, by Theorems 3.3 and 4.2, the problem (5.2) has a unique time $2 \pi$-periodic solution

$$
\tilde{x} \in \mathrm{PC}_{2 \pi}\left((0, \infty), L^{2}(\Omega)\right) \cap C^{2+v, 1+\frac{v}{2}}\left(\bar{\Omega} \times \mathbb{R}^{+}\right)
$$

which exponentially attracts $x\left(z, t ; x_{0}\right)$ in $L^{2}(\Omega)$.

Competing interests

The authors declare that they have no competing interests.

\section{Authors' contributions}

All the authors contributed equally and significantly in writing this paper. All authors read and approved the final manuscript. 


\section{Author details}

'Department of Mathematics, Shanghai Jiao Tong University, Shanghai, 200240, P.R. China. ${ }^{2}$ School of Mathematical Sciences, Fudan University, Shanghai, 200433, P.R. China.

\section{Acknowledgements}

The authors thank the referees very much for their careful reading and helpful suggestions.

$J$ Liang and TJ Xiao acknowledge support from NSFC (No. 11171210, 11271082, 11371095). H Yang acknowledges support from NSF of Gansu province (No. 1308RJZA217) and China Postdoctoral Science Foundation Funded Project (No. BR0710008).

\section{Received: 6 November 2015 Accepted: 1 December 2015 Published online: 15 December 2015}

\section{References}

1. Abada, N, Benchohra, M, Hammouche, H: Existence and controllability results for nondensely defined impulsive semilinear functional differential inclusions. J. Differ. Equ. 246, 3834-3863 (2009)

2. Amann, $\mathrm{H}$ : Periodic solutions of semilinear parabolic equations. In: Cesari, L, Weinberger, R (eds.) Nonlinear Analysis: A Collection of Papers in Honor of Erich H. Rothe, pp. 1-29. Academic Press, New York (1978)

3. Benchohra, M, Henderson, J, Ntouyas, S: In: Impulsive Differential Equations and Inclusions. Comtemp. Math. Appl., vol. 2. Hindawi Publ. Corp., Cairo (2006)

4. Cuevas, C, Sepúlveda, A, Soto, H: Almost periodic and pseudo-almost periodic solutions to fractional differential and integro-differential equations. Appl. Math. Comput. 218, 1735-1745 (2011)

5. Cuevas, C, Pierri, M, Sepúlveda, A: Weighted S-asymptotically $\omega$-periodic solutions of a class of fractional differential equations. Adv. Differ. Equ. 2011, Article ID 584874 (2011)

6. De la Sen, M: About robust stability of Caputo linear fractional dynamic systems with time delays through fixed point theory. Fixed Point Theory Appl. 2011, Article ID 867932 (2011)

7. De la Sen, M: Total stability properties based on fixed point theory for a class of hybrid dynamic systems. Fixed Point Theory Appl. 2009, Article ID 826438 (2009)

8. De la Sen, M: About robust stability of dynamic systems with time delays through fixed point theory. Fixed Point Theory Appl. 2008, Article ID 480187 (2008)

9. Diagana, T: Almost periodic solutions to some second-order nonautonomous differential equations. Proc. Am. Math. Soc. 140, 279-289 (2012)

10. Diagana, T: Pseudo-almost periodic solutions for some classes of nonautonomous partial evolution equations. J. Franklin Inst. 348, 2082-2098 (2011)

11. Diagana, T: The existence of a weighted mean for almost periodic functions. Nonlinear Anal. 74, 4269-4273 (2011)

12. Henry, D: Geometric Theory of Semilinear Parabolic Equations. Lecture Notes in Math., vol. 840. Springer, New York (1981)

13. Lakshmikantham, V, Bainov, D, Simeonov, P: Theory of Impulsive Differential Equations. World Scientific, Singapore (1989)

14. Li, YX: Existence and uniqueness of periodic solution for a class of semilinear evolution equations. J. Math. Anal. Appl. 349, 226-234 (2009)

15. Li, YX: Existence and asymptotic stability of periodic solution for evolution equations with delays. J. Funct. Anal. 261 1309-1324 (2011)

16. Liang, J, Liu, JH, Xiao, TJ: Nonlocal impulsive problems for nonlinear differential equations in Banach spaces. Math Comput. Model. 49, 798-804 (2009)

17. Liang, J, Liu, JH, Xiao, TJ: Periodic solutions of delay impulsive differential equations. Nonlinear Anal. 74, 6835-6842 (2011)

18. Liu, JH: Periodic solutions of infinite delay evolution equations. J. Math. Anal. Appl. 247, 644-727 (2000)

19. Liu, JH: Bounded and periodic solutions of infinite delay evolution equations. J. Math. Anal. Appl. 286, 705-712 (2003)

20. Liu, XZ: Impulsive stabilization and applications to population growth models. Rocky Mt. J. Math. 25, 381-395 (1995)

21. Machado, JA, Ravichandran, C, Rivero, M, Trujillo, JJ: Controllability results for impulsive mixed-type functional integro-differential evolution equations with nonlocal conditions. Fixed Point Theory Appl. 2013, 66 (2013)

22. Mophou, GM, N'Guérékata, GM: Existence of Antiperiodic Solutions to Semilinear Evolution Equations in Intermediate Banach Spaces. In: Advances in Interdisciplinary Mathematical Research, Springer Proc. Math. Stat, vol. 37, pp. 133-139. Springer, New York (2013)

23. Mophou, GM: Existence and uniqueness of mild solutions to impulsive fractional differential equations. Nonlinear Anal. 72, 1604-1615 (2010)

24. Mophou, GM: Almost automorphic solutions of some semilinear fractional differential equations. Int. J. Evol. Equ. 5(1), 109-115 (2010)

25. Pazy, A: Semigroup of Linear Operator and Applications to Partial Differential Equations. Springer, Berlin (1983)

26. Temam, R: Infinite-Dimensional Dynamical Systems in Mechanics and Physics, 2nd edn. Springer, New York (1997)

27. Xiao, TJ, Liang, J: The Cauchy Problem for Higher Order Abstract Differential Equations. Lecture Notes in Math. vol. 1701. Springer, Berlin (1998)

28. Ahmed, N: Optimal impulsive control for impulsive systems in Banach spaces. Int. J. Differ. Equ. 1, 37-52 (2000)

29. Ahmed, N: Some remarks on the dynamics of impulsive systems in Banach spaces. Dyn. Contin. Discrete Impuls. Syst., Ser. A Math. Anal. 8, 261-274 (2001)

30. Ahmed, N: Existence of optimal controls for a general class of impulsive systems on Banach spaces. SIAM J. Control Optim. 42, 669-685 (2003)

31. Barreira, L, Valls, C: Lyapunov regularity of impulsive differential equations. J. Differ. Equ. 249, 1596-1619 (2010) 